\title{
BIFURCATIONS AND INVARIANT SETS IN A CLASS OF TWO-DIMENSIONAL ENDOMORPHISMS
}

\author{
DJELLIT ILHAM ${ }^{1, *}$, FAKROUNE YAMINA ${ }^{1}$ AND SELMANI WISSAME ${ }^{2}$ \\ ${ }^{1}$ Laboratory of Mathematics, Dynamics and Modelization, University Badji Mokhtar, Annaba-Algeria \\ ${ }^{2}$ University 20 Aout 1955, Skikda-Algeria \\ *Corresponding author: ilhem.djelit@univ-annaba.dz
}

\begin{abstract}
Several endomorphisms of the plane have been constructed by simple maps. We study the dynamics occuring in one of them, which is rich in global bifurcations. The invariants sets are stable manifolds of saddle type points or cycles, as well as closed curves issued from Hopf bifurcations. The present paper focuses some bifurcations related with attractors or basins which produce other attractors which coexist with invariant sets.
\end{abstract}

\section{INTRODUCTION}

Multistable systems, i.e. systems with a large number of coexisting stable systems, are very common in nature. They are subject of increasing interest in the last two decades. It has been the discovery of multiple stable states in many systems (Agarwal in [1]; Arecchi et al. in [2]; Djellit and Soula in [4]) that triggered the research in many other fields. Multistable behaviors were found in discrete systems (Hénon in [9]; Ikeda in [10]; Grebogi et al. in [7]; Feudel et al. in [6]; Lorenz in [11]).

We present and explain numerical results illustrating the mechanism of bifurcations that occurs in a typical dynamical system relative to "coupled-uncoupled" two-dimensional map. Because the non-unique dynamics associated with the degree of the nonlinearity leads to a real interest and a large richness of the

Received 2019-03-25; accepted 2019-04-22; published 2019-07-01.

2010 Mathematics Subject Classification. 37G10, 37J20, 37J15, 37D10.

Key words and phrases. bifurcation; endomorphism; attractor.

(C)2019 Authors retain the copyrights of their papers, and all open access articles are distributed under the terms of the Creative Commons Attribution License. 
bifurcations situations, and interesting phenomena are uncovered, due in essence to the presence of invariant sets. This can have a profound effect on our understanding of the dynamical behavior. We provide numerical evidence and show some mechanisms associated with the appearance and disappearance of closed invariant curves.

Consider this dynamical system generated by a family of two-dimensional continuous noninvertible maps $T_{a, b}$ defined by :

$$
T_{a, b}:\left\{\begin{array}{l}
x^{\prime}=y \\
y^{\prime}=a y-b x+x^{2}
\end{array}\right.
$$

For $a=1$ and $b$ real parameter, this map was already studied by Razafimandimby [14] and in Clerc and Hartmann [3]. They considered the influence domains of stable singularities of the quadratic endomorphism and they studied self-intersections of the unstable set in the form of loops. This fact has fundamental and known consequences in bifurcations theory particularly in global bifurcations and chaos. Such bifurcations governing the route to chaos have been extensively studied since 1975 in $[8,12]$.

Many of chaotic behaviors that are observed in dynamical systems are intimately associated with the presence of homoclinic and/or heteroclinic points of maps. Contact bifurcations may correspond to homoclinic and heteroclinic bifurcations, critical and /or invariant curves are useful for interpreting such problems. Several papers have shown the importance of critical curves in the bifurcations of basins, for example Gumowski and Mira [8] who have developed the role of these curves in bifurcations from simply connected basin to nonconnected basin, Ferchichi et al. in [5] and Mira et al. in [13] have studied bifurcations of type Simply connected basin to multiply connected basin. These basic bifurcations result from the contact of a basin boundary with a critical curve segment or an attracting set leading to modifications of the basin.

We study an endomorphism using both analytic bifurcation theory and numerical methods. The dynamics involves various transitions by bifurcations. The analysis of the dynamics of this model as a function of three parameters $(a, b$,and $n)$ is not an easy task. The particular case, with $a=1$ fixed, as a function of $(b, n=2)$ has been studied in Ref. [3]. There it has been shown that for the map $T$, the bifurcations of the model and route to chaos could be well determined.

The planar system is the following:

$$
T_{a, b, n}:\left\{\begin{array}{l}
x^{\prime}=y \\
y^{\prime}=a y+f_{n}(x)
\end{array}\right.
$$

where $x, y$ are real variables, $a$ and $b$ are real parameters. $T_{a, b, n}$ has a nonconstant Jacobian determinant $\operatorname{det} J=-f_{n}^{\prime}(x)$.

These systems $T_{a, b, n}$ which become uncoupled when $a=0$, are of fundamental importance in dynamical systems. Two main tools are used by developing the method of critical curves and incorporating invariant 
curves : two well-established methods. We display invariant sets for $n=2$, and 4 and we examine their properties. We mainly focused on this research area, the identification and verification of some properties of such maps.

First we consider the structure of the one-dimensional endomorphism of the $n$-degree polynomial

$$
f_{n}(x)=x^{n}-b x=x\left(x^{n-1}-b\right)
$$

where $f_{n}(x)$ is assumed to depend continuously on the real parameter $b$.

For $n$ even, $n=2 k, k \geqslant 1$, the fixed points of $f_{n}$ are $: x=0$ a stable node for $\left.b \in\right]-1 ; 1\left[\right.$ and $x_{1}=(b+1)^{\frac{1}{n-1}}$ stable node for $b \in] \frac{n+1}{n-1} ;-1[$.

For $n=2 k+1$, the equation $f_{n}(x)=x$ admits a unique trivial solution $x=0$ for $b \leq-1$ : and for $b>-1$; we have three solutions $x=0 ; x_{1}=(b+1)^{\frac{1}{n-1}}$, and $x_{2}=-(b+1)^{\frac{1}{n-1}}$.

For $n=2$, the two-dimensional endomorphism (2) has some symmetry property and possesses at most 2 fixed points depending upon the parameter values. We describe a specific family of bifurcations in a region of real parameter plane $(a, b)$ for which the mappings were expected to have simple dynamics. We compute the first few bifurcation curves in this family and we study the bifurcation diagram which consists to these bifurcation curves in the parameter $(a, b)$ plane. The Figures 1.( $a, b, c, d, e, f)$ give the parameter value for which at least one fixed point is attractive (blue domain corresponding to the value 1). More generally, these figures give the existence domains of a stable cycle of order $k$ of which is given by the upper colored squares. The black regions $(k=15)$ corresponds to the existence of bounded iterated sequences. These figures are typical of maps with dominating odd or even degree terms. We can recognize on the diagram period doubling bifurcations, and spring area communication according to Mira [13]. The bifurcation structure is a " boxwithin-a-box" type, as is well known infinitely many periodic are opened by fold bifurcations and are closed by homoclinic bifurcations by the intriguing " box-within-a-box " bifurcation structure. When $n$ is even, there is an interesting passage from symmetric parameter diagram to non symmetric parameter diagram (clearly visible for the existence domain related with fixed points of color blue). And we can remark that for $n$ even or odd, the diagrams are quite similar and then we can argue that some properties are automatically deduced. For $n=2$, the two existence domains associated with the two fixed points are symmetric with respect to a black line located in the frontier to each domain, for $n$ even or odd, the existence domains of different k-cycles shrink. For $n=3$, the two domains are superimposed and symmetric to the line $a=0$. This case $(n$ odd $)$ is treated separately in an other paper.

Despite the simplicity of this map, the concept "coupled-uncoupled" is central to our interest and subject. So, in the following we shall focus our attention on the description of the bifurcations which are expected to occur as we vary the parameters along the bifurcation path indicated in Fig. 1(a). 


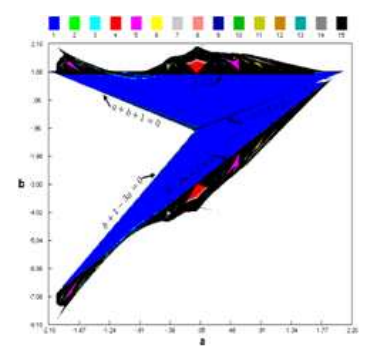

(a) bifurcation structure $\mathrm{n}=2$

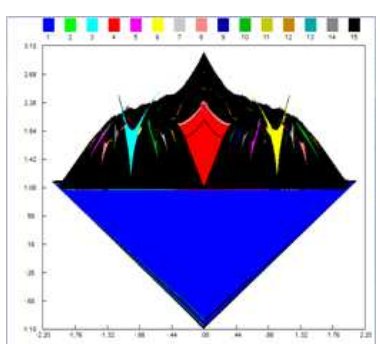

(b) bifurcation structure $n=3$

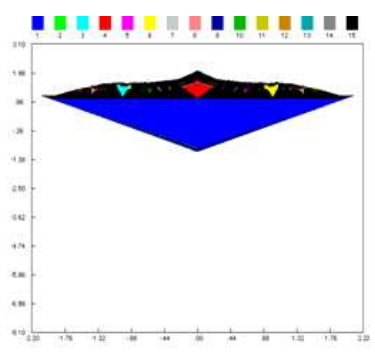

(e) bifurcation structure $n=6$
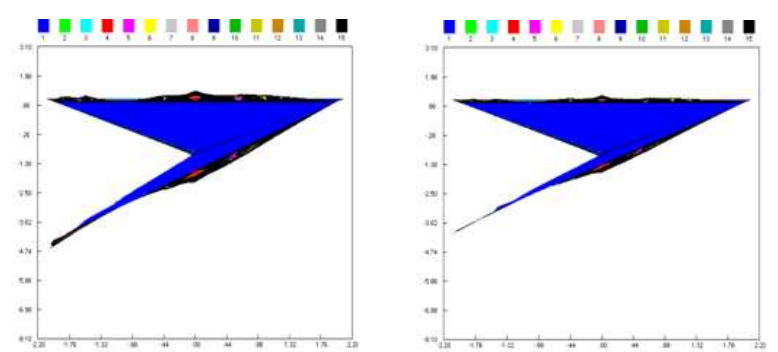

(c) bifurcation structure $\mathrm{n}=4$

(d) bifurcation structure $\mathrm{n}=5$

Figure 1. Bifurcation structure for $n$ even and odd values.

This paper intends to give such a study, particularly to consider the map (2) with $n=2$. Therefore, it is structured in the following way. In Section 2 we introduce the language used in $[12,13]$; to analyze the system, and give some properties. Section 3 gives some results concerning the uncoupled map and the main bifurcations. Section 4 illustrates properties of invariant curves and connected-nonconnected bifurcation basins.

\section{Symmetry AND FUndamental PRoperties}

First we analyse the dynamics for the case $T=T_{a, b, 2}$ of the form

$$
T_{a, b, 2}(x, y)=\left(y, a y+f_{2}(x)\right)
$$

with $f_{2}(x)=x^{2}-b x, a$ and $b$ are real parameters. Let us consider the case $f_{2}(x)$ a quadratic polynomial $(n=2)$. We know some results which enable us to detect, predict, determine cycles and fixed points, and locate bifurcation curves in the parameter plane.

The fixed points and basic bifurcations of $T_{a, b, 2}$ were analyzed, they are solutions obtained by a trivial manipulation with $x^{\prime}=x$ and $y^{\prime}=y$ : Besides the trivial solution $O(0 ; 0)$, we can observe that further fixed point $P(1+b-a, 1+b-a)$ exists if $a \neq 1+b$.

In this section we focus our attention on bifurcations playing an important role in the dynamics, those happening for $a \neq 0$ and $b \neq 0$. We can easily state the following proposition. 
Proposition 2.1. : If $b=-1+a$, then $O(0 ; 0)$ is the unique fixed point of the map $T_{a, b, 2}$ defined by (1). The parameter curve $\Lambda_{(1)_{0}}: b=-1+a$ is the transcritical bifurcation curve such that the two fixed points $O$ and $P$ exist, and are symmetric with respect to the parameter curve.

Consider the variable change that moves the point $P$ to coincide with the origin as follows

$\left\{\begin{array}{l}x=x^{*}+1+b-a \\ y=y^{*}+1+b-a\end{array}\right.$.

After injection of this change in $T\left(=T_{a, b, 2}\right)$ and some simple simplification, we have

$$
\left\{\begin{array}{l}
x^{*}=y^{*} \\
y=a y^{*}-x^{*} * x^{*}-(2 a-b-2) x^{*}
\end{array}\right.
$$

We put $b^{*}=2 a-b-2$, and $a^{*}=a, T_{a, b, 2}$ becomes $T_{a^{*}, b^{*}, 2}$.

To simplify the notation, in the following we shall write $T^{*}$ instead of $T_{a^{*}, b^{*}, 2}$. This new map $T^{*}$ has two fixed points $(0,0)$ which corresponds to $P$ for $T$ and $P^{*}\left(1+b^{*}-a^{*}, 1+b^{*}-a^{*}\right)$ which corresponds to the trivial solution for $T$. These two maps $T$ and $T^{*}$ change their fixed points.

Let $L: \mathbb{R}^{2} \rightarrow \mathbb{R}^{2}$

$$
(x, y)_{(a, b)} \rightarrow\left(x^{*}, y^{*}\right)_{\left(a^{*}, b^{*}\right)}=(x-(1+b-a), y-(1+b-a))_{(a, 2 a-b-2)}
$$

Proposition 2.2. $L$ is symmetric by respect to $\Delta$, or $L \circ T=T^{*} \circ L$.

Corollary 2.1. If $P$ is a fixed point of $T$, then $L(P)$ is a fixed point of $T^{*}$.

Corollary 2.2. The parametric curve $\Lambda_{(1)_{0}}: b=-1+a$ is invariant by $L$.

The fixed point $P(1+b-a, 1+b-a)$ with the parametric vector $(a, b)$ is associated with the fixed point $O(0,0)_{\left(a^{*}, b^{*}\right)}$ related to parametric vector $\left(a^{*}, b^{*}\right)=(a, 2 a-b-2)$. Thus both fixed point $\mathrm{O}$ and $\mathrm{P}$ can undergo identical sets of bifurcations in parameter space. We can choose the parameters so that one fixed point experiences all the bifurcations, while the other has none. It suffices then to study the nature and bifurcations of $O(0,0)$; those of $P$ are deduced automatically.

$$
J=\left|\begin{array}{cc}
0 & 1 \\
-b+2 x & a
\end{array}\right|
$$

We consider now the conditions of local stability of the fixed point $O(0 ; 0)$, in terms of the parameters of the map $T$.

With $J_{(0,0)}=\left|\begin{array}{cc}0 & 1 \\ -b & a\end{array}\right|$, is Jacobian matrix of $T$ in $O(0 ; 0)$ which has two eigenvalues $\lambda_{1}=\frac{a}{2}+\sqrt{\frac{a^{2}}{4}-b}$; $\lambda_{2}=\frac{a}{2}-\sqrt{\frac{a^{2}}{4}-b}$. We can conclude for $a^{2}-4 b>0$ with considering $b<\frac{a^{2}}{4}$ :

Proposition 2.3. If $1+a+b=0$ or $1+b+3 a=0$, the map $T$ undergoes a flip bifurcation at the fixed point $O(0,0)$ or $P(1+b-a, 1+b-a)$, respectively. 
Proof. Consider the square map $T^{2}($ the second iterate of $T): T^{2}(x ; y)=T\left(y, a y-b x+x^{2}\right)=(a y-b x+$ $\left.x^{2}, a\left(a y-b x+x^{2}\right)-b y+y^{2}\right)$.

Fixed points of $T^{2}$ are computed by considering $T^{2}(x, y)=(x, y)$, we obtain then : $a y-b x+x^{2}=x$ and $a\left(a y-b x+x^{2}\right)-b y+y^{2}=y$. We put from the first equation $y=\left((1+b) x-x^{2}\right) / a$, and by replacing $y$ with its value in the second equation we obtain a quartic polynomial of $x$. After simplification of the two fixed points, we have this quadratic polynomial : $Q(x)=x^{2}-(1+a+b) x+a(1+a+b)$. Roots of $Q(x)$ are the abscissa of the two points of the cycle of order $2: x_{1,2}=((1+a+b) \pm \sqrt{(1+a+b)(1+b-3 a)}) / 2$ and the ordinates are : $y_{1}=x_{2} ; y_{2}=x_{1}$.

The sufficient condition for the existence of the 2-cycle is given by $(1+a+b)(1+b-3 a)>0$. If $(1+a+$ $b)(1+b-3 a)=0$ we have a flip bifurcation.

Let us denote :

$\Lambda_{1}=\left\{\left(\begin{array}{l}a \\ b\end{array}\right) / 1+a+b=0\right\}$ and $\Lambda_{1}^{\prime}=\left\{\left(\begin{array}{l}a \\ b\end{array}\right) / 1+b-3 a=0\right\}$

If $1+a+b=0$ we have $x_{1}=x_{2}=0$, this flip bifurcation line $\Lambda_{1}$ is associated with the fixed point $O(0,0)$.

If $1+b-3 a=0$ we have $x_{1}=x_{2}=2 a$, this flip bifurcation line $\Lambda_{1}^{\prime}$ is associated with the other fixed point $P(1+b-a, 1+b-a)$.

Also the two curves $\Lambda_{1}$ and $\Lambda_{1}^{\prime}$ are clearly visible in Figure 1.(a).

Proposition 2.4. The map $T$ undergoes discrete Hopf bifurcations at the fixed point $O(0,0)$ for $a=2$ cos $\left(\alpha_{k}\right)$ and $b=1$.

Proof. We obtain the parameter values related to Hopf bifurcations for the fixed point $O(0,0)$ of focus type if we put the eigenvalues $\lambda_{1}=\exp \left(i \alpha_{k}\right)$ and $\lambda_{2}=\exp \left(-i \alpha_{k}\right)$ with $\alpha_{k}=\frac{2 \pi}{k} ; k \in \mathbb{N}$.

Proposition 2.5. The parametric line $b-2 a+3=0$ is the symmetric of the line $\Delta: b=1$ with respect to the line $\Lambda_{(1)_{0}}: b-a+1=0$.

This line $\Delta^{\prime}: b-2 a+3=0$ is associated with the fixed point $P$ and plays a key role for global bifurcations. Similarly for $\Delta$ which is associated with the trivial fixed point $O$ for global bifurcations.

Proof. With a simple computation we obtain this line.

From Fig1.(a), we can see that the stability domain of the trivial fixed point $O=(0,0)$ is represented by a blue triangle, bounded by the transcritical bifurcation curve $\Lambda_{(1)_{0}}: b-a+1=0$, the flip bifurcation curve $1+a+b=0$ and the Hopf bifurcation curve $\Delta: b=1$. And for the fixed point $P(1+b-a, 1+b-a)$ another blue triangle is associated, bounded by the transcritical bifurcation curve $\Delta^{\prime}: b-a+1=0$, the flip bifurcation curve $1+b+3 a=0$ and the Hopf bifurcation curve $\Delta^{\prime}: b-2 a+3=0$ 


\section{UNCOUPLED MAP PROPERTIES}

For $a=0$, the second iterate of the map, i.e. $T^{2}$ is a uncoupled map because $T^{2}(x ; y)=T\left(y,-b x+x^{2}\right)=$ $\left(-b x+x^{2},-b y+y^{2}\right)=\left(f_{2}(x), f_{2}(y)\right)$ is with separate variables.Then, the dynamics of $T$ is associated with those of $f_{2}$ and their bifurcations also are strictly related. If we consider then $f_{2}(x)=-b x+x^{2}$, its derivative is $f_{2}^{\prime}(x)=-b+2 x$. For $-3<b<-1: f_{2}$ has two points, the trivial point $x=0$ which is unstable with an eigenvalue equal to $b$, and the other point $b+1$ stable and its eigenvalue is equal to $b+2$.

Also $T$ has two fixed points $O=(0 ; 0)$ et $P=(b+1 ; b+1)$. The first point is a saddle with eigenvalues $\lambda_{1,2}= \pm \sqrt{-b}$. The second point $P$ has two eigenvalues $\lambda_{1,2}= \pm \sqrt{b+2}$ is a stable star-node for $\left.b \in\right]-2 ;-1[$ ; and a stable focus for $b \in]-3 ;-2\left[\right.$. T possesses a 2 -cycle which is a saddle $C_{T}^{2}=\{(0 ; b+1) ;(b+1 ; 0)\} ;$ with eigenvalues $b ; b+2$.

For $b=-1 ; f_{2}(x)$ has a unique fixed point $x=0$; such that $f_{2}^{\prime}(0)=1$; that means that $b=-1$ is a transcritical bifurcation value.

For $b=-3$; the fixed point $x=0$ is still unstable, and the other point $b+1$ has an eigenvalue equal to -1 ; $b=-3$ is a flip bifurcation value.

For $-1-\sqrt{6}<b<-3$; the second point $b+1$ becomes unstable and gives rise to a stable $2-$ cycle $C_{f_{2}}^{2}$ : $\left\{x_{1} ; x_{2}\right\}=\left\{\frac{1}{2}[b-1+\sqrt{(b-1)(b+3)}] ; \frac{1}{2}[b-1-\sqrt{(b-1)(b+3)}]\right\}$, with an eigenvalue equal to $1-(b-$ 1) $(b+3)$.

T has 4-cycle $C_{T}^{4}:\left\{\left(x_{1} ; x_{2}\right) ;\left(x_{2} ; x_{2}\right) ;\left(x_{2} ; x_{1}\right) ;\left(x_{1} ; x_{1}\right)\right\}$.

For $b=-1-\sqrt{6}$; the $2-$ cycle $C_{f_{2}}^{2}:\left\{x_{1} ; x_{2}\right\}$ of $f_{2}(x)$ becomes unstable since $f_{2}^{2 \prime}(x)=-1$, and a $4-$ cycle $C_{f_{2}}^{4}:\left\{x_{3} ; x_{4} ; x_{5} ; x_{6}\right\}$ appears.

Two stable nodes 8 -cycles of homogene type are generated by the 4 -cycle : $C_{T, 1}^{8}:\left\{T^{i}\left(x_{3} ; x_{3}\right) ; i=1, \ldots, 8\right\}$ $; C_{T, 2}^{8}:\left\{T^{i}\left(x_{4} ; x_{3}\right) ; i=1, \ldots, 8\right\}$ these two 8 -cycles have attraction basins of rectangular form and disjoints. The combinaison of the $2-$ cycle $C_{f_{2}}^{2}:\left\{x_{1} ; x_{2}\right\}$ with the $4-$ cycle $C_{f_{2}}^{4}:\left\{x_{3} ; x_{4} ; x_{5} ; x_{6}\right\}$ leads to the creation of four 8-cycles of mixed saddle type. And two others are given by the combinaison with the two fixed points such that $C_{T, 3}^{8}:\left\{T^{i}\left(x_{1} ; x_{3}\right) ; i=1, \ldots, 8\right\} ; C_{T, 4}^{8}:\left\{T^{i}\left(x_{2} ; x_{3}\right) ; i=1, \ldots, 8\right\} ; C_{T, 5}^{8}:\left\{T^{i}\left(0 ; x_{3}\right) ; i=1, \ldots, 8\right\} ;$ $C_{T, 6}^{8}:\left\{T^{i}\left(1+b ; x_{3}\right) ; i=1, \ldots, 8\right\}$.

We remark the emergence of a decreasing sequence of the parameter values of $b:\left\{b_{1} ; b_{2} ; b_{3}, \ldots\right\}$ which correspond to flip bifurcation values. These values of $b$ tend towards $b_{1}$ such the attractor becomes a Cantor set.

Table of the parameter values of $b: f_{2}(x)=-b x+x^{2}$ :

\begin{tabular}{|c|c|c|c|c|c|c|}
\hline $\mathrm{n}$ & 1 & 2 & 3 & 4 & 5 & 6 \\
\hline$b_{n}$ & -3 & -3.44931 & -3.54402 & -3.56437 & -3.56875 & -3.56994 \\
\hline
\end{tabular}


We give the position of cycles for the map $T$ and precisely cycles which are generated by an infinite sequence of flip bifurcations and provocate chaos. The two fixed points $O$ and $P$ can be stable inside a given region of the space of the parameters of the map, and can lose stability via a Hopf bifurcation, as well as a transcritical bifurcation or a flip bifurcation. This last situation is reported in the qualitative Fig. 2.( $a, b, c)$ for $b<0$, when $O$ is unstable and $P$ a stable point.

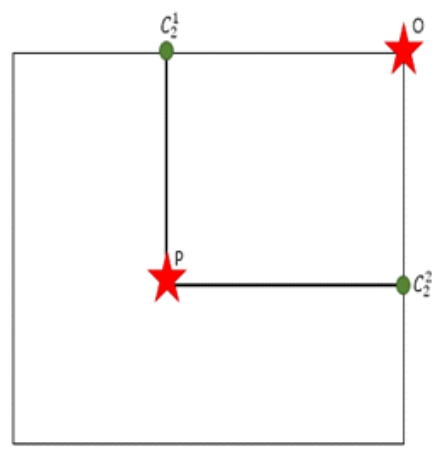

(a) $b<0 ; O$ and $P$ the two fixed points and $C_{T}^{2}=\left\{C_{2}^{1} ; C_{2}^{2}\right\}=\{(0 ; b+$ $1) ;(b+1 ; 0)\}$

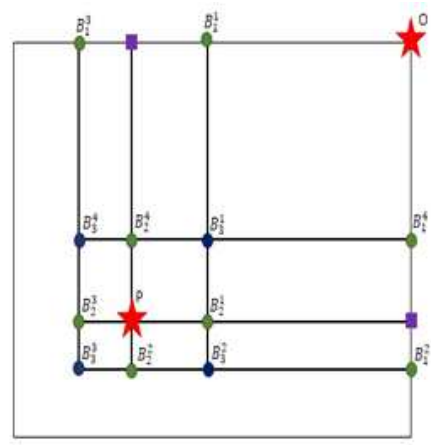

(b) Different cycles of order 4

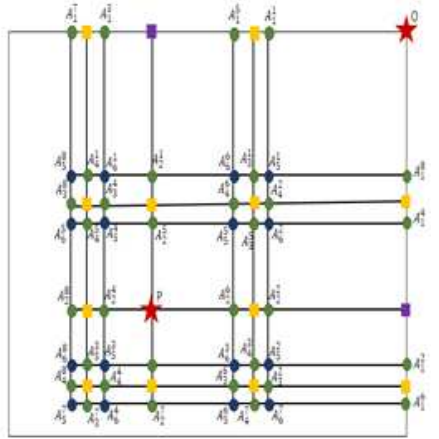

(c) Different cycles of order 8

Figure 2. Different cycles for $b<0$.

Other pecular properties that can be deduced from the properties of the one-dimensional map $f_{2}(x)$ for the endomorphism $T$ when $a=0$ concern critical curves $L C_{-1}, L C, L C_{1}, L C_{2}$ which are given here by

$$
\left(L C_{-1}: x=s_{-1} ; \text { where } s_{-1}=\frac{b}{2} \text { is the critical point of rank } 0 \text { of } f_{2}(x)\right)
$$

and

$$
\left(\begin{array}{c}
L C: y=s_{0}=f_{2}\left(s_{-1}\right) ; L C_{1}: x=s_{0}=-\frac{b^{2}}{4} \\
L C_{2}: y=s_{1} ; L C_{3}: x=s_{1}
\end{array}\right) \text { with } s_{k}=f_{2}\left(s_{k-1}\right) \text { of } \operatorname{rank}(k+1)
$$

For $b=-2$, the critical points $s_{-1}, s_{0}, s_{1}$ are equal, $b<-2$, the interval $I=\left[s_{0}, s_{1}\right]$ is an absorbing interval containing an attracting set, but for $b>-2$, the interval $I=\left[s_{0}, s_{1}\right]$ is not an absorbing interval.

For selected values of the parameter $b$ and for $n=2$, the coexistence of many different local attractors opens an important question on their behavior, and the problem of the delimitation of the boundaries of their basins which are made up of rectangles. In Figures 3.(a,b), the immediate basin (containing attractors) is formed by disjoint rectangles bounded by segment of the critical curves which are straight lines parallel to the coordinates axes, and issued from the critical points of $f_{2}(x)$. Studying these basins help in understanding the ways of multistability formation. We refer to [13] for its complete description.

The bifurcation curves related to the two values of $b=1$ and $b=-3.7$ correspond to homoclinic bifurcation curves for the fixed points $O$ or $P$ and determine also the end of the range in which the quadratic shape of $f_{2}(x)$ plays no role. We can see an explosion of straight lines of different colors bounding rectangular basins. 


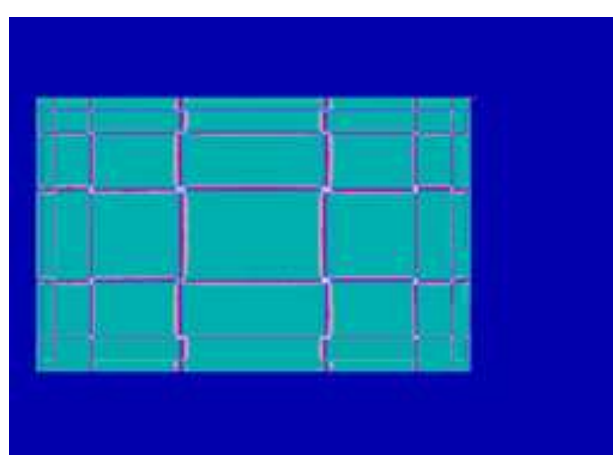

(a) $a=0 ; b=1$

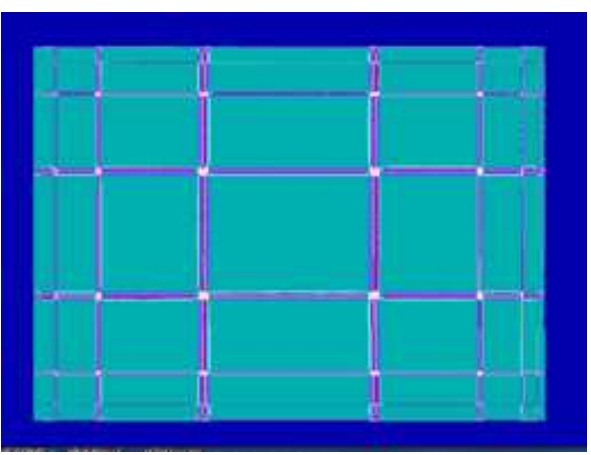

(b) $\mathrm{a}=0 ; \mathrm{b}=-3.7$

FiguRE 3. Basins and critical curves.

\section{BASINS AND INVARIANT CURVES}

We present some results of numerical simulations and discuss their implications. We have to explain the creation of two invariant curves, one issued from a fixed point of saddle type, constitutes the basin boundary of the second one which is an attracting closed curve. The phase portrait of the quadratic endomorphism $T$ is studied by constructing regular invariant curves associated to saddles and Hopf bifurcation. In this section, the regions of parameters chosen for numerical experiments contain parametric values which are not arbitrary but characteristic values with focus on selected properties. Thus, in the following we shall focus our attention on the description of the bifurcations which are expected to occur as we vary the parameters $a$ close to 2 in (a) and (b) and -2 in (c) and (d) and $b$ close to the value 1 . Since $b>0$, the fixed point $O$ is stable, inside its attraction basin undergoes a Hopf bifurcation and a little closed curve exists inside the basin, together with a closed stable manifoldof the saddle point $P$.

The following figures show the corresponding basin structure of $T$ for $n=2$. Figures 4. $(a, b, c, d, e, f)$ represent the existing attractors (the two fixed points $O ; P$, an invariant closed curve $(I C C)$ around the fixed point $O$ after a Hopf bifurcation) and the stable manifold emanating from the saddle point. In Figs 4. $(a, b) a \simeq 2, b \simeq 1\left(\lambda_{1}=\lambda_{2}=1\right)$, the two fixed points exist, $P$ is a saddle and its invariant stable manifold delimits the basin of $O$ which has undergone a Hopf bifurcation when $a$ uncreases from 1.7 to 1.9. The two curves are closed and invariant. In Figs 4. $(c, d), a \simeq-2, b=1\left(\lambda_{1}=\lambda_{2}=-1\right)$, the fixed point $O$ undergoes the flip bifurcation $\Lambda_{1}(1+b+a=0)$ and the 2 -cycle is still merged with $O$ which gives again a $I C C$. In Figs 4. $(e, f)$, and for $n=4$ we have nearly the same behavior with invariant curves associated with the fixed points. We observe that small changes in the location of the critical curve $L C_{-1}$ which has some effects on the properties of the attractors, and it may cause remarkable asymmetries in the structure of the basins, which can only be detected from the global properties of the studied model. 


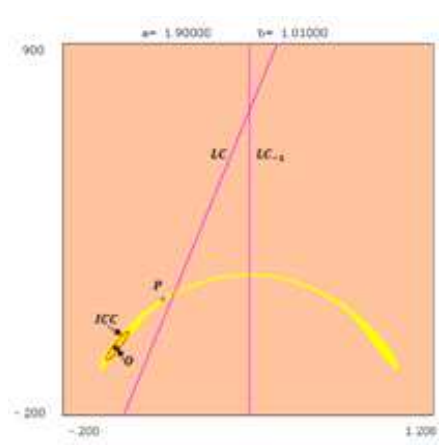

(a) Invariant closed curve in a yellow basin $n=2$

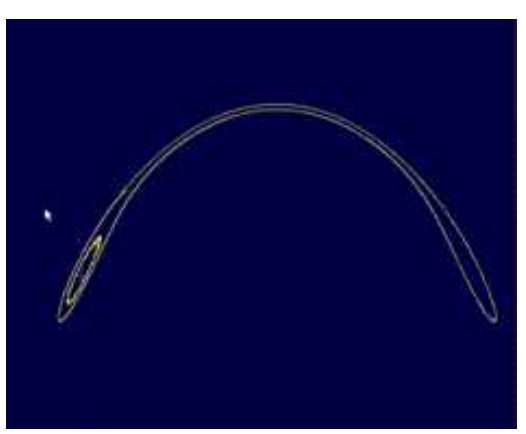

(b) Only invariant curves are displayed $n=2, x=\{-0.36, . ., 1\}$, $y=\{-0.28, . ., 0.15\}$

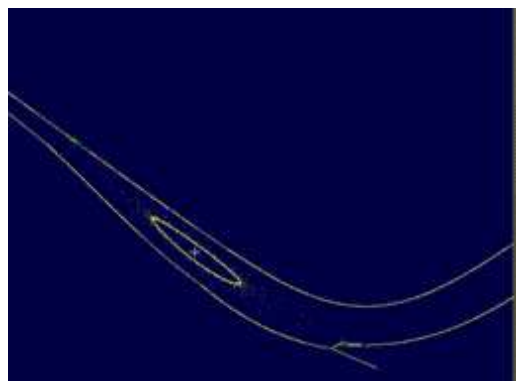

(d) Only invariant curves are displayed $n=4, x=\{-1, . ., 1\}$, $y=\{-0.28, . ., 1\}$ (e) $a>0$

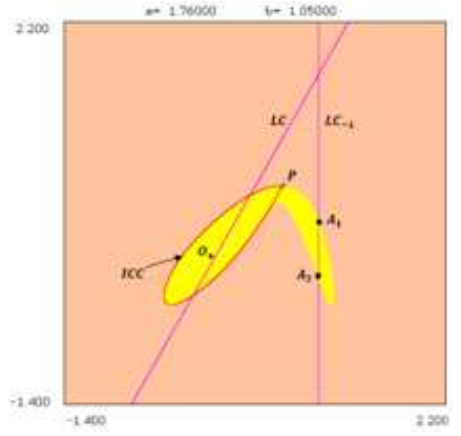

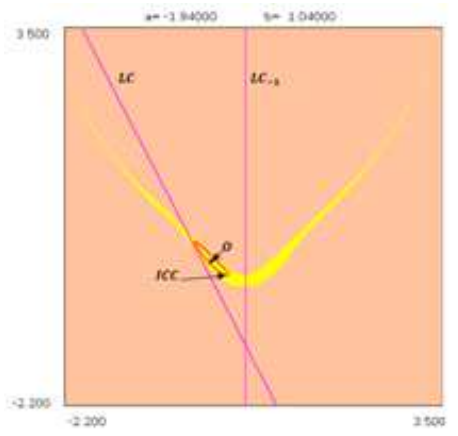

(c) Invariant closed curve in a yellow basin $n=4$

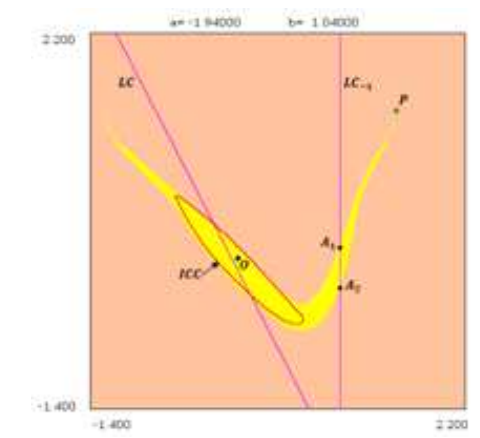

(f) $a<0$

Figure 4. Bounded basin delimited by the invariant stable manifold and an ICC around the stable fixed point.

Other invariant sets for $n=2$ (and similarly for $n$ even) can be estimated for $T^{2}$. These invariant sets are obtained by iterating invariant lines in the immediate bounded basin. The case $a=-2$ is very interesting, because we can put in evidence the existence of such sets. The special character of this kind of sets has been already observed in uncoupled maps. To illustrate the idea, we put $L_{1}: y_{2}=\alpha x_{2}+\beta, x_{2}$ and $y_{2}$ are the second iterates of $x$ and $y$. We obtain then $a\left(a y-b x+x^{2}\right)-b y+y^{2}=\alpha\left(a y-b x+x^{2}\right)+\beta$. By virtue of invariance of $L_{1}$ by $T(y=\alpha x+\beta)$, we have :

For all $x,\left(a+\alpha^{2}-\alpha\right) x^{2}+\left(a^{2} \alpha-a b+2 \alpha \beta-a \alpha^{2}\right) x+\left(a^{2} \beta-b \beta+\beta^{2}-a \alpha \beta-\beta\right)=0$.

Then $\quad a+\alpha^{2}-\alpha=0 ; \beta\left(-b+\beta-a \alpha^{2}-1\right)=0$ and $-a b+2 \alpha \beta-a \alpha^{3}=0$

For $a=-2$, we have $L_{1}: y=-x+b-1$. If we take $b=1+\varepsilon$, then $L_{1}: y=-x+\varepsilon$

The iterates $T\left(L_{1}\right)$ are also invariant because $T^{2}\left(T\left(L_{1}\right)\right)=T\left(T^{2}\left(L_{1}\right)\right)=T\left(L_{1}\right)$.

$T\left(L_{1}\right)$ is a parabola whose equation is $y=\frac{x^{2}}{\alpha^{2}}+\left(a-\frac{b}{\alpha}-\frac{2 \beta}{\alpha^{2}}\right) x+\frac{\beta}{\alpha}\left(b+\frac{\beta}{\alpha}\right)=x^{2}-b x-b+1$ 
If we take $\beta=0$, we have $L_{2}: y=0, L_{3}: y=x$.

Besides the elements seen thus far, there is another particularity in the dynamics of $T$. The strong dependence on the parameters causes a rich variety of complex patterns on the plane and gives rise to different kinds of basins. Taking into account the complexity of the matter and its nature, the study of these phenomena can be carried out only via the association of numerical investigations guided by fundamental considerations that can be found in [13]. The endomorphism (2) with $a \neq 0$ is of type $\left(Z_{0}-Z_{2}\right)$, whose three first critical curves $L C_{-1}, L C, L C_{1}^{\prime}$ are given here by

$$
\left(L C_{-1}:\left\{(x, y) / x=\frac{b}{2}\right\}\right)
$$

and

$$
\left(\begin{array}{c}
L C: T\left(L C_{-1}\right)=\left\{(x, y) / y=a x-\frac{b^{2}}{4}\right\} \\
L C_{1}: \quad\left\{(x, y) / y=\frac{1}{a^{2}} x^{2}+\left(a+\frac{b^{2}}{2 a^{2}}-\frac{b}{a}\right) x+\frac{b^{4}}{16 a^{2}}-\frac{b^{3}}{4 a}\right\}
\end{array}\right)
$$

The inverses $T_{ \pm}^{-1}$ are given by:

$$
T_{ \pm}^{-1}(x, y)=\left\{\begin{array}{l}
x=\frac{b}{2} \pm \frac{1}{2} \sqrt{b^{2}+4(y-a x)} \\
y=x
\end{array}\right.
$$

Attractors constitute an interesting set of study by themselves. Their attraction basins are split in two equal parts by $L C_{-1}$.

To understand the behavior of the map when $a$ is close to -2 , we follow the evolution in the phase plane when we vary the parameter $b \quad(b \approx-5.87, b=0.95)$.

All these situations have been shown in [13] proposed by Mira et al. for a wide variety of reasons and different models, in all they incorporate nonlinear terms and quadratic nonlinearities. In most cases, the basin of attraction undergoes some changes. The geometric structure of the basin is occurring for particular choices of the parameters.

Recent works dealing with cases of multiple attractors in noninvertible maps have highlighted how noninvertibility can become a source of bifurcations and complex structure in the basins of attraction. However, the global phenomena and the complex structures of the basins shown here are due to the both sets which are critical and invariant sets.

For $a=-2$, the critical curves $L C_{-1}, L C, L C_{1}$ and $L C_{2}$ delimit the attractor that occupies the whole basin. The invariant lines of $T$ and $T^{2}$ respectively $(y=x$ and $y=-x+1)$ contain fixed points $(0,0)$ and $(5,5)$ for $y=x$ and the two points of the $2-\operatorname{cycle}\{(2,-1) ;(-1,2)\}$ for $y=-x+1$ (see Fig. 6.).

\section{Conclusion}

This family of polynomial maps with $n=2$ is symmetric with respect to the curve $L C_{-1}$. And the bifurcations shown in the different sections are related to this curve, but we can observe that basins become asymmetric if $n=2 k, k>1$. A variety of interesting properties in this family are pointed out, including degenerate and 


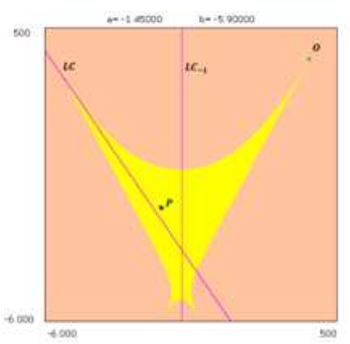

(a) Connected basin

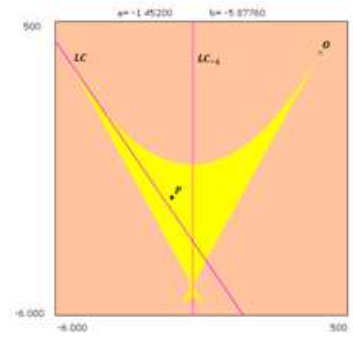

(b) Bifurcation basin to nonconnected basin

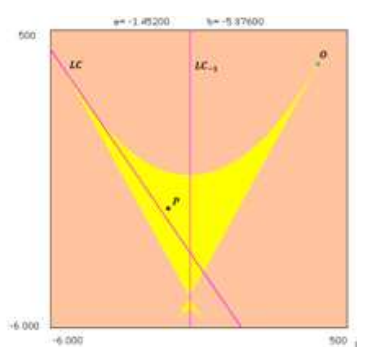

(c) Nonconnected basin, $b<$

0

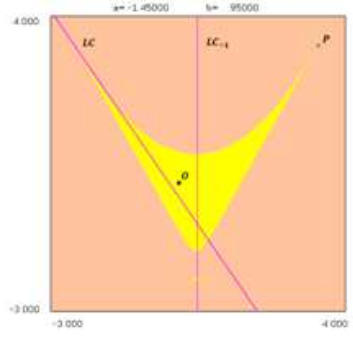

(d) Nonconnected basin, $b>$ 0

Figure 5. The evolution of basin in the phase plane.

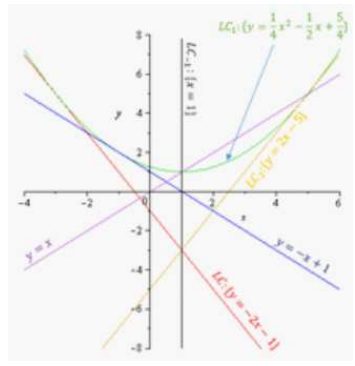

Figure 6. Critical and invariant sets for $a=-2$.

global bifurcations. According to the bifurcation diagram shown in Fig.1(a) and by exploiting the relation between invariant sets and critical curves, we have considered four Parametric points $(a, b)$ which are of two or three codimension bifurcation points, $(-2,1),(-2,-7),(2,1)$ and $(0,-1)$. The two first points are of two-degree of complexity and the two last points are of three-degree of complexity, the behavior of the solutions is mainly determined by the invariant sets and critical curves. We are not trying to show the details of the bifurcation mechanisms leading to the dynamic situations described above for $n=2 k, k>1$, however, we believe that they have identical behavior to those observed in Secs. 2 and 3, and display the same structure associated with the basin bifurcation connected-nonconnected described in Sec. 4, even if they occur in a very narrow interval of values of the parameter $b$.

\section{REFERENCES}

[1] G. S. Agarwal, Existence of multistability in systems with complex order parameters, Phys. Rev. A, 26 (1982), 888-891.

[2] F. T. Arecchi, R. Meucci, G. Puccioni and and J. Tredicce, Experimental evidence of subharmonic bifurcations, multistability, and turbulence in a q-switched gas laser, Phys. Rev. Lett., 49 (1982), 1217-1220.

[3] R. L. Clerc and C. Hartmann, Invariant manifolds of separable discrete dynamic systems, Dynamics Days, La Jolla, California, (1982).

[4] I. Djellit and Y. Soula, On riddled sets and bifurcations of chaotic attractors, Appl. Math. Sci., 1 (13) (2007), 603-614. 
[5] M. R. Ferchichi, I. Djellit and J. C. Sprott, Broken symmetry in modified Lorenz model, Int. J. Dyn. Syst. Differential Equations, 5 (2) (2015), 136-148.

[6] U. Feudel and C. Grebogi, Multistability and the control of complexity, Chaos, 7 (1997), 597-604.

[7] C. Grebogi, E. Kostelich, E. Ott and J. A. Yorke, Multi-dimensioned intertwined basin boundaries: Basin structure of the kicked double rotor, Physica D, 25 (1987), 347-360.

[8] I. Gumowski, C. Mira, Dynamique chaotique, Ed. Cepadues, Toulouse, (1980).

[9] M. Henon, A two-dimensional mapping with a strange attractor, Commun. Math. Phys., 50, (1976), 69-77.

[10] K. Ikeda, Multiple-valued stationary state and its instability of the transmitted light by a ring cavity system, Opt. Commun., 30 (1976), 257-261.

[11] H. W. Lorenz, Multiple attractors, complex basin boundaries, and transient motion in deterministic economic systems, in Dynamic Economic Models and Optimal Control, ed. Feichtinger, G. (Elsevier), (1992).

[12] C. Mira, Chaotic dynamics, World Scientific, (1987).

[13] C. Mira, L. Gardini, A. Barugola and J. C. Cathala, Chaotic dynamics in two-dimensional non invertible maps, World Scientific, (1996).

[14] B. Razafimandimby, Domaine d'influence de certaines singularités stables d'un endomophisme de $\mathbb{R}^{2}$, Thèse de 3ème cycle, Univ. Paul Sabatier, Toulouse, (1981). 